\title{
Control of ring lasers by means of coupled cavities
}

\section{Buchhave, Preben; Abitan, Haim; Tidemand-Lichtenberg, Peter}

\section{Published in:}

Nonlinear Optics: Materials, Fundamentals, and Applications

Link to article, DOI:

10.1109/NLO.2000.883639

Publication date:

2000

Document Version

Publisher's PDF, also known as Version of record

Link back to DTU Orbit

Citation (APA):

Buchhave, P., Abitan, H., \& Tidemand-Lichtenberg, P. (2000). Control of ring lasers by means of coupled cavities. In Nonlinear Optics: Materials, Fundamentals, and Applications (pp. 254-256).

https://doi.org/10.1109/NLO.2000.883639

\section{General rights}

Copyright and moral rights for the publications made accessible in the public portal are retained by the authors and/or other copyright owners and it is a condition of accessing publications that users recognise and abide by the legal requirements associated with these rights.

- Users may download and print one copy of any publication from the public portal for the purpose of private study or research.

- You may not further distribute the material or use it for any profit-making activity or commercial gain

- You may freely distribute the URL identifying the publication in the public portal

If you believe that this document breaches copyright please contact us providing details, and we will remove access to the work immediately and investigate your claim. 


\title{
Control of ring lasers by means of coupled cavities
}

\author{
Preben Buchhave, Haim Abitan, and Peter Tidemand-Lichtenberg \\ Dept. of Physics, Technical University of Denamrk, Buildg. 309, DTU DK-2800 Lyngby, Denmark \\ e-mail: phu@fivik.du.dk
}

\author{
Abstract: \\ Variable phase coupling to an external ring is used to control a unidirectional ring laser. The \\ observed behavior of the coupled rings is explained theoretically. \\ (C)1999 Optical Society of America \\ OCIS Codes: $190.2620,140.3560$
}

Coupling of optical cavities offers a means of controlling the properties of one cavity (e.g. a laser) by making adjustments to another, external cavity. In this contribution we consider a unidirectional ring laser (bow-tie laser) coupled to an external ring cavity. Using different configurations we can control the outcoupling from the ring laser thereby influencing the threshold and operating point of the laser. This may be used to match the output coupling strength to the internal losses in the laser, optimizing the power output. It is also possible to choose a circulating power level that provides the best balance between the passive losses and a nonlinear loss such as e.g. conversion to the second harmonic.

We have found experimentally that by quickly changing the phase of the feedback from the external ring it is possible to Q-switch the ring laser. Also, at certain values of the phase of the feedback in the external ring, instabilities in the total system occur and oscillations arise in the ring laser.

The theoretical description involves the solution of a set of transcendental nonlinear equations, one for the laser, one for the second harmonic generation and one for the output coupling. The coupling is controlled by the transmission properties of the coupled FP-ring. We believe the facilities of modern PC-based mathematics programs offer new possibilities for quickly and conveniently solving these equations and obtain information on the complex behavior of coupled nonlinear resonators that have so far not been available.

We consider first the general case of two coupled rings shown in Fig. 1. The equation for the laser is

$$
P_{p, s d l}=\frac{h v_{s d l}}{\tau_{c}} \frac{l_{e}}{c}\left(\frac{2 P_{i}}{h v_{d p l}}+\frac{V_{u}}{\sigma l \tau}\right) \text {. }
$$

This expression gives the pump power, $P_{p, s d l}$, needed to produce a circulating laser power, $P_{i}$. Here $\sigma$ denotes the stimulated emission cross section, $h v_{\text {sdl }}$ the pump photon energy, $h v_{d p l}$ the laser photon energy and $c$ the speed of light. $l$ is the crystal length, $l_{c}$ is the geometric cavity length, $V$ is the mode volume and $V_{a}=\left(l / l_{c}\right) V$ is the volume of the mode inside the active medium. We also define an effective optical path length $l_{e}=l_{c}+(n-1) l$, where $n$ is the index of refraction of the active medium. Further $\tau_{c}=l_{e} /(\alpha c)$ is the lifetime of the laser photons in the cavity and $\tau$ is the excited state lifetime. $\alpha$ is the total distributed loss per pass containing contributions from absorption, mirror transmissions and second harmonic conversion. We denote explicitly the total internal losses including absorption, scattering and mirror transmission by $\alpha_{i}$ and the conversion to second harmonic by $\alpha_{S H C}=\left(P_{c 4}-P_{c 3}\right) / P_{c 4}$. In relation to Fig. 1 we can identify $P_{i}$ with $\left(P_{c 3}+P_{c 4}\right) / 2$, and $P_{p, s d l}$ with $p p$. 


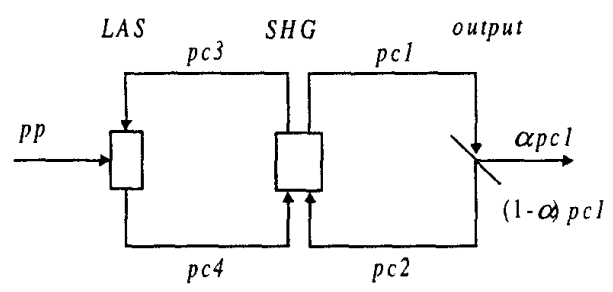

Fig. 1. Principle of coupled ring cavities.

For the coupling to the second ring we consider two cases: Coupling through nonlinear conversion to the second harmonic and coupling through dielectric mirrors.

For the coupling through the conversion to the second harmonic we use the relations [1]:

$P_{c l}=P_{t o t} \tanh ^{2}\left[g z_{0}+g z_{c}\right]$

$P_{c 3}=P_{t o t} \operatorname{sech}^{2}\left[g z_{0}+g z_{c}\right]$

where

$g z_{0}=\tanh ^{-1}\left[\sqrt{\frac{P_{c 2}}{P_{t o t}}}\right]$ and $P_{t o t}=P_{c 2}+P_{c 4}$.

The gain is proportional to the square root of the total power, $g=c_{2} \sqrt{P_{t o t}}$, where $c_{2}$ is a materials constant characterizing the actual SHG-crystal.

Inserting $g z_{0}$ we can write

$$
\begin{aligned}
& P_{c 1}=P_{t o t} \tanh ^{2}\left[\tanh ^{-1}\left[\sqrt{\frac{P_{c 2}}{P_{t o t}}}\right]+g z_{c}\right] \\
& P_{c 3}=P_{t o t} \operatorname{sech}^{2}\left[\tanh ^{-1}\left[\sqrt{\frac{P_{c 2}}{P_{t o t}}}\right]+g z_{c}\right] .
\end{aligned}
$$

In case of ordinary output coupling by a dielectric mirror we have $P_{o u t}=R P_{c 1}$ and $P_{c 2}=(1-R) P_{c 1}$, where $R$ is the power reflectivity of the output mirror.

However, in coupled resonators, the reflectivities of the dielectric coupling mirrors depend on the field incident from the return beam. In general, the complex electric field reflectivity of any mirror in the ring depends on all the others:

$$
\frac{E_{r}}{E_{i}}=f\left(r_{1}, r_{2}, r_{3}, r_{4}, r_{5}, r_{6}, \Phi_{1}, \Phi_{2}\right) \text {, }
$$

where $r_{i}$ is the reflectivity of the i'th mirror and $\Phi_{j}$ is the total phase in the $\mathrm{j}^{\prime}$ th ring. The functional form depends on the configuration. We thus have to solve the coupled field equations including the variable mirror reflectivity to see the effect of the feedback. When the rings include nonlinear effects, like the laser gain, second harmonic generation or sum/difference generation, we must solve a set of simultaneous transcendental equations. This has been done by means of a PC-version mathematics program.

We have specifically considered a bow-tie unidirectional ring laser coupled to a triangular ring as shown in Fig. 2. At first we consider the coupled rings without the KTP second harmonic crystal. Experimentally, we vary the phase by means of the manual control box, which in turn varies the apparent reflectivity of the 
mirrors M5 and M6. We can for instance cause the laser to go below threshold by adjusting the phase of the feedback loop in such a way that the reflectivities of M5 and M6 become high. We can also adjust the phase of the return path such that the output from the mirror $M 7$ is optimized.

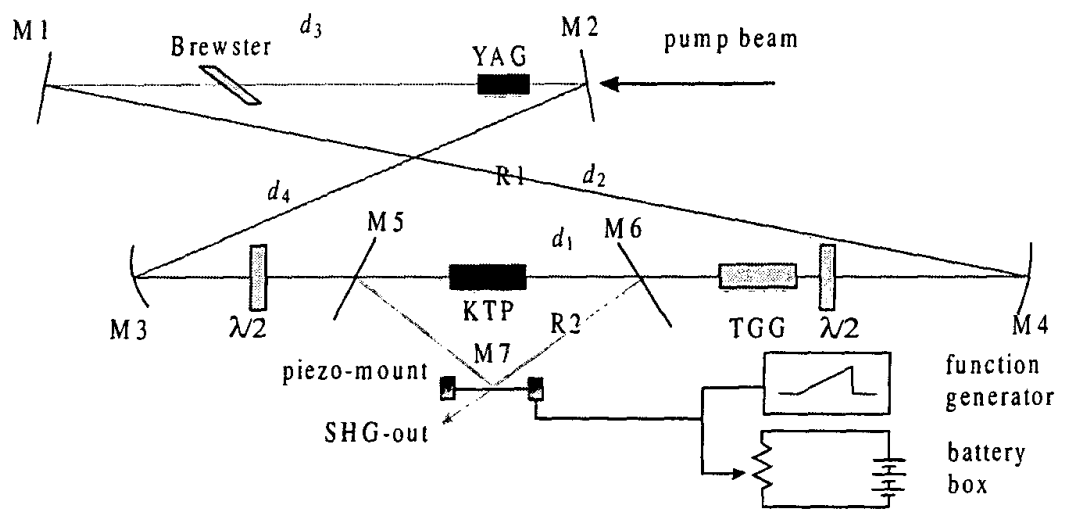

Fig. 2. Unidirectionl ring laser coupled to a triangular ring cavity.

If the phase is made to change quickly, the ring laser is observed to Q-switch just by means of the variable phase control. We have also observed oscillations in the system at certain steady-state adjustments of the phase of ring $\mathrm{R} 2$ relative to the phase of ring $\mathrm{R} 1$. The waveform of the oscillations are similar to the pattern derived in [2].

In a further development we consider the coupled rings with the KTP-crystal inserted. The SH generation now depends on the phase of the return sh-signal. By varying the piezo-mirror, we can measure the effect of a phase variation in the feedback.

\section{REFERENCES}

J. A. Armstrong, N. Bloembergen, J. Ducuing and P. S. Pershan, "Interaction between light waves in a nonlinear dielectric", Phys. Rev. 127, 1918-1939 (1962).

C. M. Savage and D. F. Walls, "Optical chaos in second-harmonic generation", Optics Acta 30, 557-61 (1983). 